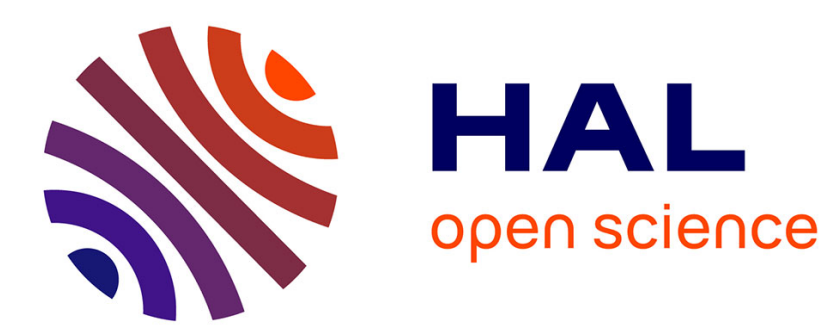

\title{
Microcanonical Lanczos Method
}

Xenophon Zotos

\section{To cite this version:}

Xenophon Zotos. Microcanonical Lanczos Method. Philosophical Magazine, 2006, 86 (17-18), pp.25912601. 10.1080/14786430500227830 . hal-00513552

\section{HAL Id: hal-00513552 \\ https://hal.science/hal-00513552}

Submitted on 1 Sep 2010

HAL is a multi-disciplinary open access archive for the deposit and dissemination of scientific research documents, whether they are published or not. The documents may come from teaching and research institutions in France or abroad, or from public or private research centers.
L'archive ouverte pluridisciplinaire HAL, est destinée au dépôt et à la diffusion de documents scientifiques de niveau recherche, publiés ou non, émanant des établissements d'enseignement et de recherche français ou étrangers, des laboratoires publics ou privés. 


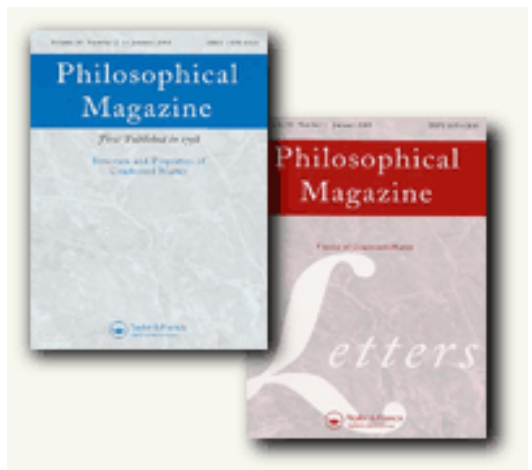

\section{Microcanonical Lanczos Method}

\begin{tabular}{|r|l|}
\hline Journal: & Philosophical Magazine \& Philosophical Magazine Letters \\
\hline Manuscript ID: & TPHM-05-Feb-0039 \\
\hline Journal Selection: & Philosophical Magazine \\
\hline Date Submitted by the \\
Author: & 15 -Feb-2005 \\
\hline Complete List of Authors: & Zotos, Xenophon; University of Crete, Physics \\
\hline Keywords: & $\begin{array}{l}\text { magnetic insulators, electronic transport, numerical simulation, } \\
\text { statistical physics }\end{array}$ \\
\hline Keywords (user supplied): & \\
\hline &
\end{tabular}

\section{今) ScholaroNE \\ Manuscript Central}




\section{Microcanonical Lanczos Method}

\section{ZOTOS}

\section{Department of Physics, University of Crete} and Foundation for Research and Technology-Hellas, P. O. Box 2208, 71003 Heraklion, Crete, Greece

In this paper I will discuss the development of a novel numerical simulation method based on the Lanczos technique and the microcanonical statistical ensemble. It allows the evaluation of finite temperature dynamic correlations for quantum many body systems on lattices larger than hitherto possible. To demonstrate the applicability of the method, I will review a series of recently obtained results for one dimensional spin and electronic models.

Keywords: numerical simulation, quantum, dynamic correlation, statistical ensembles 


\section{Motivation}

An outstanding open problem in quantum many body physics is the development of reliable numerical simulation methods for the evaluation of finite temperature dynamic correlations. These correlations are intimately related, via the fluctuation-dissipation theorem, to transport quantities and thus they are of direct interest in the interpretation of experiments [1]. Furthermore, exact numerical results are indispensable for the verification of analytical approaches and the exploration of new concepts.

Regarding the domain of applicability of these methods, there is a great experimental and theoretical interest in the last few years on the physics of novel materials as the high-temperature superconductors, the manganites or the quasi-one dimensional compounds.

Theoretically, the physics of these systems is often formulated and explored using prototype electronic or magnetic models on a lattice as the Hubbard, Heisenberg and $t-J$ Hamiltonian. A dynamic correlation at a finite temperature $T=1 / \beta$ is given by,

$$
\left\langle X_{q}(t) X_{-q}(0)\right\rangle
$$

where $X_{q}$ is the $q$-component of an observable, e.g. a density, current, spin magnetization operator. Using a spectral representation, we obtain,

$$
\left\langle X_{q}(t) X_{-q}(0)\right\rangle=\sum_{m, n} p_{n}\left|\left\langle n\left|X_{q}\right| m\right\rangle\right|^{2} e^{i\left(\epsilon_{n}-\epsilon_{m}\right) t},
$$

with $\mid n>, \epsilon_{n}$ the eigenstates, eigenvalues of the Hamiltonian and $p_{n}$ the corresponding Boltzmann weights. With respect to experiment, the dynanic structure factor, relevant e.g. to neutron scattering experiments is given by,

$$
S(q, \omega)=\int_{-\infty}^{+\infty} d t e^{i \omega t}\left\langle X_{q}(t) X_{-q}(0)\right\rangle
$$

and the susceptibility, related to absorption, by,

$$
\chi(q, \omega)=i \int_{-\infty}^{+\infty} d t e^{i z t}\left\langle\left[X_{q}(t), X_{-q}(0)\right]\right\rangle, \quad z=\omega+i \eta .
$$

In principle, the numerical evaluation of (2) is straightforward. By diagonalizing the Hamiltonian matrix using standard codes (e.g. the Eispack library) one can obtain all the eigenvalues and eigenstates and thus have a complete description of the dynamic correlation. The problem however is 
the size of the matrix that can be numerically diagonalized, which is limited to a dimension of a few thousand states. Considering the size of the Hilbert space $D$, we find that for an $S=1 / 2$ spin system it grows as $D \sim 2^{L}(L$ is the number of spins), while for a Hubbard type electronic model with 4 states per site (empty, up, down, doubly occupied) it grows as $D \sim 4^{L}$, in other words exponentially fast in both cases. Thus, the size of the systems that can be studied is typically limited to $L \sim 18$ spins and $L \sim 10$ sites for tight binding electronic systems. Of course, the eventual symmetries, e.g. translational, spin, of the system can be used to reduce the size of matrices, but the reduction is not crucial as the size of the matrices is exponentially large. Here, we should point out that, in general, the geometry of the lattice and the complexity of the interaction/hopping terms in the Hamiltonian does not enter the counting of the dimension of the Hilbert space and thus the effectiveness of a numerical diagonalization method depends only on the number of states per site.

\section{2. $\mathrm{T}=0$ Lanczos method}

A way out of this counting restriction is offered by the Lanczos diagonalization method, essentially a projection technique, that allows the evaluation of the extreme eigenvalues and eigenvectors of far larger matrices, of the order of a few million states [2].

Once the ground state eigenfunction $|0\rangle$ has been calculated by a first Lanczos procedure, a second Lanczos procedure provides a continued fraction expansion of the structure factor $[3,4]$,

$$
\begin{aligned}
S(q, \omega) & =-2 \lim _{\eta \mapsto 0} \Im\left\langle 0\left|X_{q} \frac{1}{[z-H]} X_{-q}\right| 0\right\rangle \\
& =\frac{\left\langle 0\left|X_{q} X_{-q}\right| 0\right\rangle}{a_{0}-\frac{b_{1}^{2}}{z-a_{1}-\frac{b_{2}^{2}}{z-\ldots}}}
\end{aligned}
$$

The coefficients $a_{0}, a_{1}, b_{1}, \ldots$ are evaluated by a successive application of the Hamiltonian, starting from the ground state $|0\rangle$ acted on by $X_{q}$,

$$
\begin{aligned}
\left|f_{n+1}\right\rangle & =H\left|f_{n}\right\rangle-a_{n}\left|f_{n}\right\rangle-b_{n}^{2}\left|f_{n-1}\right\rangle \\
\left|f_{0}\right\rangle & =X_{q}|0\rangle .
\end{aligned}
$$


With the new dimensions of Hamiltonian matrices that can be analyzed, the zero temperature - ground state - spectral functions of up to $L \sim 32$ spin- $1 / 2$ and $L \sim 16$ sites electronic systems have been extensively studied. Now, the key issue of scaling as a function of lattice size can be considered, as a series of, admittedly still small, systems can be studied and the obtained results extrapolated in order to obtain the behavior at the thermodynamic limit.

So the problem posed is whether it is possible to use this twofold Lanczos procedure to evaluate dynamic correlations at finite temperatures for systems as large as it possible at zero temperature.

\section{Microcanonical Ensemble}

The answer to this question and the idea behind the 'Microcanonical Lanczos method' we propose is the equivalence of the microcanonical to the canonical statistical ensemble. In a pictorial manner it is described in the classic reference of Landau and Lifshitz [5];

It may again mentioned that, according to the basic principles of statistical physics, the result of the averaging does not depend whether it is done mechanically with respect to the exact wave function of a stationary state of the system, or statistically by means of the Gibbs distribution. The only difference is that in the former case the result is expressed in terms of the energy of the body, and in the latter case as a function of its temperature.

Thus we can replace the ground state $|0\rangle$ in the above Lanczos procedure by an appropriately chosen excited state of energy equal to the mean energy of the Hamiltonian at the desired temperature.

Besides the computational interest of this proposal it is worth pointing out that, to our knowledge, no studies of the fundamental equivalence between the microcanonical and canonical ensemble for quantum dynamic correlations exist in the literature. Thus this work is a step in numerically exploring this basic postulate of nonequilibrium statistical mechanics. Clearly, analytical studies are needed to clarify, for instance, the meaning of the microcanonical ensemble for a quantum system with dense spectrum as an average over a single quantum state (or a narrow window of states) and the finite size corrections inherent in this ensemble. 


\section{Implementation}

So the idea [6] is to replace the ground state $|0\rangle$ with a state $|\lambda\rangle$, such that,

$$
\langle H\rangle_{\beta}=\lambda=\langle\lambda|H| \lambda\rangle
$$

and thus the structure factor (3) at finite temperature $T$ in the microcanonical ensemble becomes,

$$
s(q, \omega)=\int_{-\infty}^{+\infty} d t e^{i \omega t}\left\langle\lambda\left|X_{q}(t) X_{-q}(0)\right| \lambda\right\rangle .
$$

Now the state $|\lambda\rangle$ can be constructed using a 1st Lanczos projection procedure of $M_{1}$ steps using as 'Hamiltonian' the operator,

$$
K=(H-\lambda)^{2},
$$

the 'ground state' of which is a state of energy $\lambda$. At this point we should note that it is generally easy to find the dependence of the mean energy $\lambda$ as a function of temperature $T$ for a given system, using either exact methods (e.g. Bethe ansatz for one dimensional systems) or powerful numerical techniques as the Quantum Monte Carlo or Transfer Matrix Renormalization Group that very reliably provide thermodynamic quantities. In the examples shown below, we conveniently study the high temperature limit $\beta \rightarrow 0$ that corresponds to $\lambda=0$, because of the symmetry of the spectrum.

Once the state $\lambda$ has been constructed, we can perform a 2nd Lanczos procedure of $M_{2}$ steps starting from the state $X_{q}|\lambda\rangle$, that gives a continued fraction expansion of the structure factor,

$$
\begin{aligned}
s(q, \omega) & =-2 \lim _{\eta \mapsto 0} \Im\left\langle\lambda\left|X_{q} \frac{1}{[z-H]} X_{-q}\right| \lambda\right\rangle \\
& =\frac{\left\langle\lambda\left|X_{q} X_{-q}\right| \lambda\right\rangle}{a_{0}-\frac{b_{1}^{2}}{z-a_{1}-\frac{b_{2}^{2}}{z-\ldots}}} .
\end{aligned}
$$

Now, several comments on the actual implementation of this technique are in order; first, for large systems (with exponentially dense spectrum) 
the 1st Lanczos procedure does not converge to a single eigenstate but to a superposition,

$$
|\lambda\rangle=\sum_{n} a_{n}|n\rangle,
$$

where the coefficients $\left|a_{n}\right|^{2}$ are Gaussian distributed with mean $\lambda$ and variance $\sigma$. For typical values $\left.M_{1} \sim 1000, M_{2} \sim 4000, \sigma=\sqrt{\langle} K\right\rangle \sim 0.01$. Thus, the obtained spectra have a finite frequency resolution of the order of $\sigma$.

Second, for the finite size systems that we are considering, the method works actually better at high temperatures where the spectrum is dense; thus the state $|\lambda\rangle$ is a superposition over several thousand eigenstates within a narrow window and the statistical noise minimal. Note, that in the expression (8), as we are actually using the state (11), off-diagonal terms also appear that we can show that they cancel by a random-phase argument.

Third, it is clear from the above, that we are employing a microcanonical average not over a single eigenstate but over a very narrow window of states. It is an interesting fundamental question whether, in the thermodynamic limit, the spectra obtained by the two definitions coincide and in particular what are the finite size effects.

\section{Examples}

In this section I will present a sample of recent results using the microcanonical Lanczos method, of finite temperature conductivities, e.g. spin, thermal, on one dimensional magnetic and electronic models of actual interest. The motivation of these calculations is theoretical and experimental interest in the transport properties of quasi-one dimensional compounds described by prototype models. On the one hand, it has been conjectured and now confirmed, that integrable quantum models exhibit unconventional - ballistic - transport at all temperatures [7]; on the other hand, unusually high thermal conductivities have been observed in quasi-one dimensional magnetic compounds, similar to that of metals but due to magnetic excitations [8].

The aim of the following examples, is not to give a comprehensive exposition of the investigated problems but rather a flavor of the systems that can be studied and the conducitivity spectra that can be obtained using this state of the art method.

\section{Heisenberg model}


A prototype quantum many body system is the one dimensional Heisenberg model,

$$
H=J \sum_{l=1}^{N}\left(S_{l}^{x} S_{l+1}^{x}+S_{l}^{y} S_{l+1}^{y}+\Delta S_{l}^{z} S_{l+1}^{z}\right),
$$

where $S_{l}^{\alpha}(\alpha=x, y, z)$ are spin operators on site $l$; for $\mathrm{S}=1 / 2$ it is integrable using the Bethe ansatz method.

As $S^{z}$ is conserved, using the continuity equation, we can derive the spin current $j^{z}$,

$$
\begin{aligned}
& S^{z}=\sum_{l} S_{l}^{z}, \quad \frac{\partial S_{l}^{z}}{\partial t}+\nabla j_{l}^{z}=0 \\
& j^{z}=\sum_{l} j_{l}^{z}=J \sum_{l}\left(S_{l}^{x} S_{l+1}^{y}-S_{l}^{y} S_{l+1}^{x}\right) .
\end{aligned}
$$

In the high temperature $(\beta \rightarrow 0$ limit, the normalized spin conductivity, $\sigma(\omega)$, is given by,

$$
\sigma(\omega)=\frac{\sum_{n \neq m}|<n| j^{z}|m>|^{2} \delta\left(\omega-\omega_{m n}\right)}{\left\langle j^{z 2}\right\rangle},
$$

and can be evaluated [6] using our microcanonical ensemble procedure by,

$$
\sigma(\omega) \mapsto-\lim _{\eta \mapsto 0} \frac{\Im\left\langle\lambda\left|j^{z} \frac{1}{z-H+\lambda} j^{z}\right| \lambda\right\rangle}{\pi\left\langle\lambda\left|j^{z 2}\right| \lambda\right\rangle} .
$$

Using this model, we can compare the results for the spin conductivity from a canonical ensemble calculation (summing over all Hilbert states) and a microcanonical one, for two values of the anisotropy parameter $\Delta$ as shown in Fig. 1. The lattice size of $N=20$, corresponding to a Hilbert space dimension of about 9000 states, is limiting for an exact evaluation within the canonical ensemble.

[here Fig. 1]

As shown, within the statistical noise, the comparison is very encouraging and confirms the principle of applicability of the proposed method. On the spin conductivity, for $\Delta=0.5$ it reproduces the known presence of a 'Drude' peak at zero frequency, while for $\Delta=1$ it suggests a power law behavior at low frequencies.

\section{2-leg ladder}


The second system that I will discuss is the spin-1/2 ladder depicted in Fig. 2 and described by the Hamiltonian,

$$
H=J \sum_{l=1, L}\left\{\left(S_{1, l+1} \cdot S_{1, l}+S_{2, l+1} \cdot S_{2, l}\right)+\frac{J_{\perp}}{J} S_{1, l} \cdot S_{2, l}\right\},
$$

where $J\left(J_{\perp}\right)$ is the inter-chain (intra-chain) coupling.

[here Fig. 2]

Ladder compounds like $(\mathrm{La}, \mathrm{Sr}, \mathrm{Ca}){ }_{14} \mathrm{Cu}_{24} \mathrm{O}_{41}$ are experimentally and theoretically investigated as they belong to the family of high $\mathrm{T}_{c}$ superconductors, they show a spin gap, extremely high thermal conductivities attributed to magnetic excitations and upon doping they exhibit a metalic state and superconductivity.

In Fig. 3, I show the frequency dependent thermal conductivity $\kappa(\omega)$ for a lattice of $L=14$ rungs - corresponding to a Hilbert space of about 3 million states [9]. As we can see, we obtain very smooth spectra that allow to extract the d.c. $(\omega \rightarrow 0)$ conductivity and its dependence as a function of intra-chain coupling. In particular, we can establish that the addition of the intra-chain coupling, a non-integrable perturbation to the integrable $\mathrm{S}=1 / 2$ Heisenberg model, results to a $1 / J_{\perp}$ dependence of conductivity. Notice that for the $\mathrm{S}=1 / 2$ Heisenberg model the energy current operator commutes with the Hamiltonian and thus $\kappa(\omega)$ consists purely of a zero-frequency $\delta$-function 'thermal Drude' peak.

[here Fig. 3]

Similarly, as shown in Fig. 4, by varying $\lambda$ different temperatures can indeed be studied [9], although it is important to remain in the dense, high energy region of the many-body spectrum, above the gap.

[here Fig. 4]

\section{3. $S=1$}

The next system we studied [10] is the spin-1 isotropic Heisenberg chain, a non-integrable model. The high temperature spin conductivity is shown in Fig. 5, from where we see that we are able to extract an estimate of the d.c. conductivity of the order $\sigma_{d c} \sim 1.4 \beta$.

[here Fig. 5]

From the analytically known spin susceptibility $\chi$ we thus find that the spin diffusion constant, $\mathcal{D}_{s}=\sigma_{d c} / \chi \sim 1.4 \beta / \frac{2}{3} \beta \sim 2.1$. Comparing this 
value with the one obtained by the high temperature moment method $\mathcal{D}_{s}=$ $\sqrt{2 \pi S(S+1) / 3} \sim 2.1$, we find an (probably fortuitus) excellent agreement ! Similarly, from the thermal conductivity shown in Fig. 6, [here Fig. 6] we extract a value for the thermal diffusion constant, $\mathcal{D}_{t h}=\kappa_{d c} / C \sim 7.5 \beta^{2} / \frac{4}{3} \beta^{2} \sim$ 5.6 that compares favorably (same order of magnitude) with the one obtained from the moment method

$$
\mathcal{D}_{t h}=\sqrt{\pi S(S+1) / 3} /(1-3 / 4 S(S+1)) \sim 2.3 .
$$

I should emphasize again that the obvious convergence and good quality of low frequency data is the one that allows the deduction of a good estimate for the d.c. conductivities.

\section{Hubbard model}

To show the versatility of the method, I finally show here an application on a prototype integrable electronic model, the one dimensional Hubbard chain [11]. It describes a tight binding system with electrons hopping on a lattice and interacting via a local interaction $U$ as depicted in Fig. 7. [here Fig. 7]

The Hamiltonian $H$ and current $j$ are given by,

$$
\begin{gathered}
H=-t \sum_{i, s}\left(c_{i s}^{\dagger} c_{i+1, s}+\text { h.c. }\right)+U \sum_{i} n_{i \uparrow} n_{i \downarrow}, \\
j=-t \sum_{i, s}\left(i c_{i s}^{\dagger} c_{i+1, s}+\text { h.c. }\right) .
\end{gathered}
$$

Using the microcanonical method (MCLM), the high temperature conductivity $\sigma(\omega)$, the integrated one $I(\omega)=\int_{0}^{\omega} d \omega \sigma(\omega)$ and the scaling of the zero-frequency contribution the Drude weight $D / \beta$ are shown in Fig. 8.

[here Fig. 8]

It is crucial to note that the application of the microcanonical method allows for the first time the study of large enough lattices to meaningfully scale the data. In this particular example we can thus conclude that the Drude weight goes to zero and that there are anomalously large finite size effects at low frequencies attributed to the integrability of the model. Indeed we can contrast the behavior of this gapped system with the $S=1$ chain, 
Fig. 5, also a gapped system but nonintegrable. It remains an open question whether the observed low frequency behavior scales to normal - diffusive transport with a finite d.c. conductivity in the thermodynamic limit.

Finally, I should mention that actually, the scaling in zero temperature studies turns out to be more problematic than the one at finite $T$ that I demonstrated, because the obtained spectra are far sparser (fewer transition lines) and show larger statistical fluctuations.

\section{Discussion}

Some comments are in order on the applicability, extensions and improvements of this method. First, the method works best at temperatures equivalently - energies where the spectrum is dense. Thus so far it has been used at high temperatures; problems appear at low temperatures, where the spectrum is typically sparse and in particular if there is a gap in the spectrum. Efforts are underway to improve this drawback by e.g. averaging over initial states (producing different microstates $|\lambda\rangle$ ) belonging to different $k$-subspaces.

Second, it should be emphasized that this method is very flexible as it is limited only by the dimension of the Hilbert space and not by the nature of the system, e.g. co-ordination of lattice, type of degrees of freedom. Thus studies are underway on 1D, 2D, 3D electronic/magnetic systems, clusters, quantum dots, molecular magnets. 


\section{Acknowlegments}

It is a pleasure to thank the organizers Profs. A. Gonis and N. Kioussis for inviting me to participate to this stimulating Workshop. Also, I would like to acknowledge the fundamental contribution of my friends and collaborators Profs. M. Long and P. Prelovšek to the results presented in this paper. 


\section{References}

[1] S. Doniach, E.H. Sondheimer, Green's functions for solid state physicists (London, Imperial College Press, 1998)

[2] Y. Saad, Numerical methods for large eigenvalue problems (Manchester, Manchester University Press, 1992)

[3] R. Haydock, V. Heine and M.J. Kelly, J. Phys. C5, 2845 (1972)

[4] R. Gagliano and C.A. Balseiro, Phys. Rev. Lett. 59, 2999 (1987)

[5] L.D. Landau and E.M. Lifshitz, Course of Theoretical Physics: Statistical Mechanics, 5, 377 (footnote), (Pergamon Press, London - Paris, 1959)

[6] M. Long, P. Prelovsek, S. El Shawish, J. Karadamoglou and X. Zotos, Phys. Rev. B 68, 235106 (2003)

[7] X. Zotos and P. Prelovšek, in Interacting Electrons in Low Dimensions, book series Physics and Chemistry of Materials with Low-Dimensional Structures, Kluwer Academic Publishers (2003)

[8] A.V. Sologubenko and H.R. Ott, in Interacting Electrons in Low Dimensions, book series Physics and Chemistry of Materials with Low-Dimensional Structures, Kluwer Academic Publishers (2003)

[9] X. Zotos, Phys. Rev. Lett. 92, 067202 (2004)

[10] J. Karadamoglou and X. Zotos, Phys. Rev. Lett. 93, 177203 (2004)

[11] P. Prelovsek, S. El Shawish, X. Zotos, and M. Long, Phys. Rev. B70, 205129 (2004) 

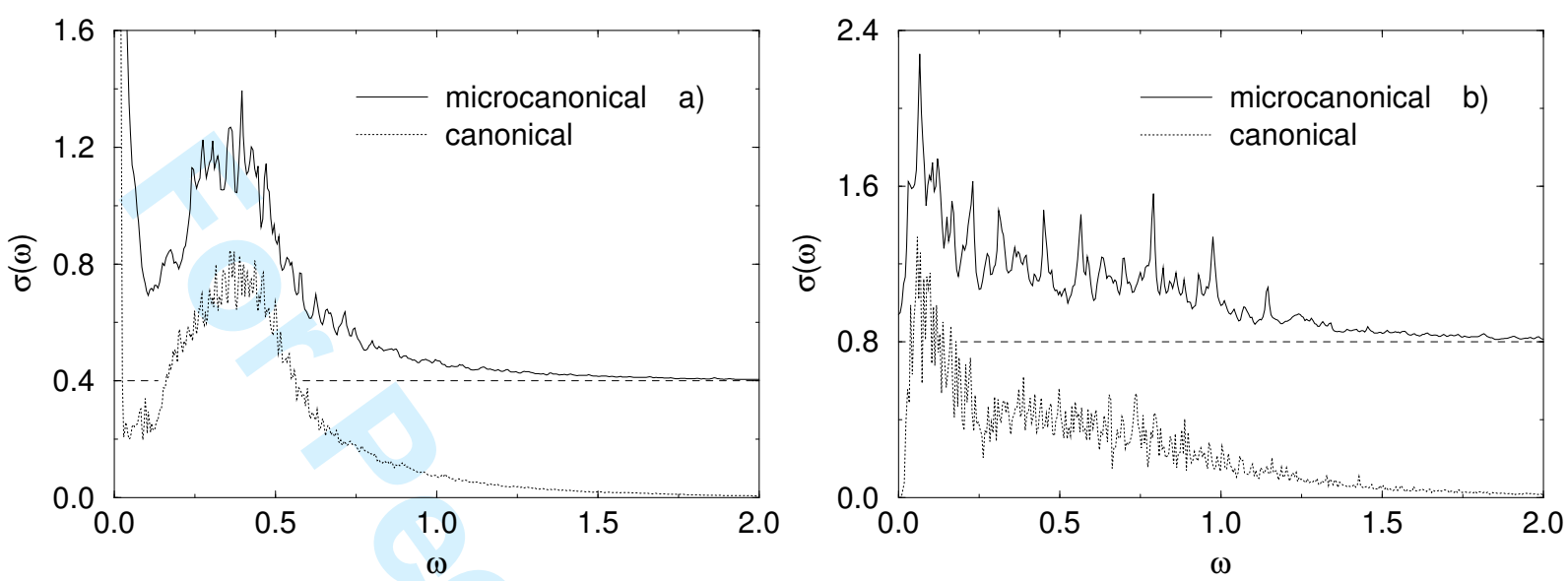

Figure 1: Microcanonical versus Canonical calculations (displaced for clarity); (a) $N=20, \Delta=0.5, \eta=0.01$, (b) $N=18, \Delta=1, \eta=0.01$.

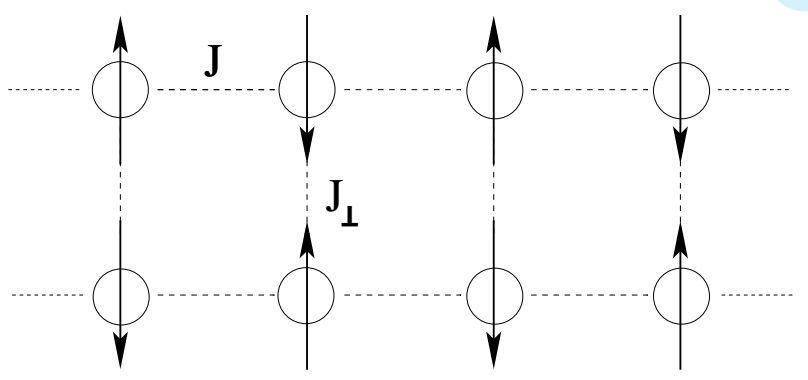

Figure 2: The spin-1/2 ladder 


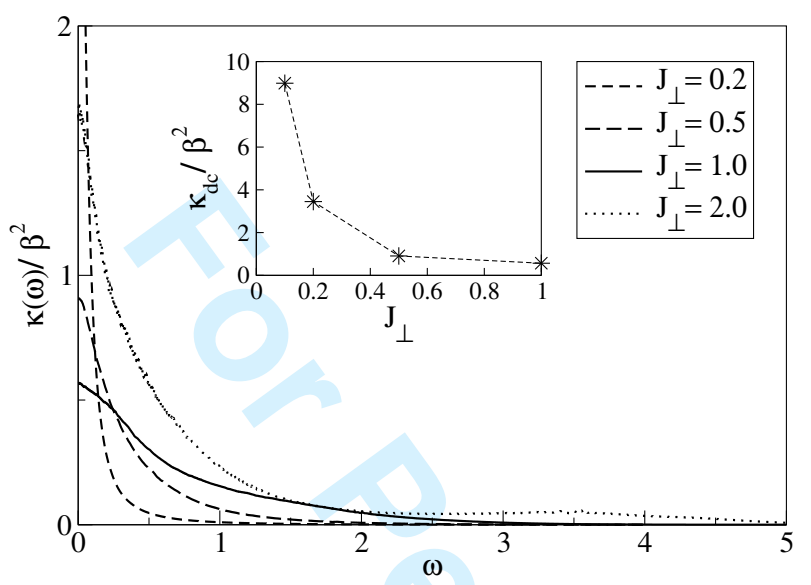

Figure 3: (a) Thermal conductivity for $L=14$ rungs as a function of $J_{\perp}$ for $\beta \rightarrow 0$. Inset: $d c-$ limit.

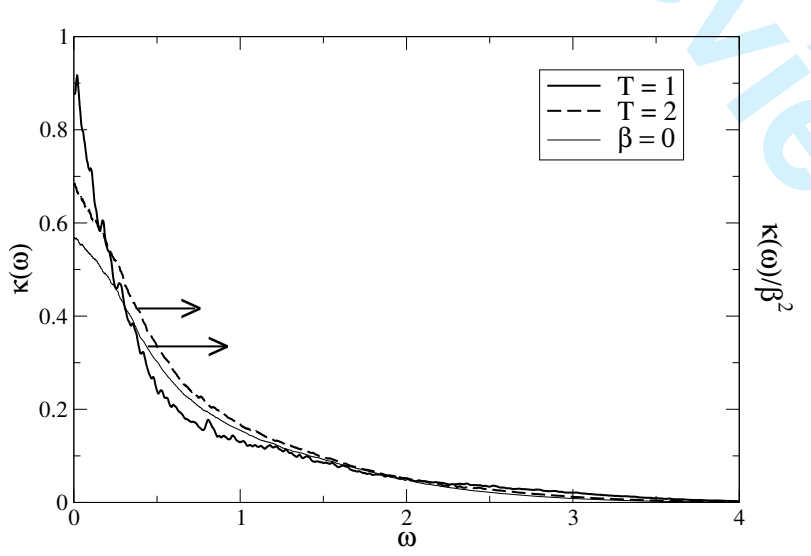

Figure 4: Thermal conductivity for $L=14$ at $T=1, T=2(\beta=0.5)$ and for comparison at $\beta \rightarrow 0$. 


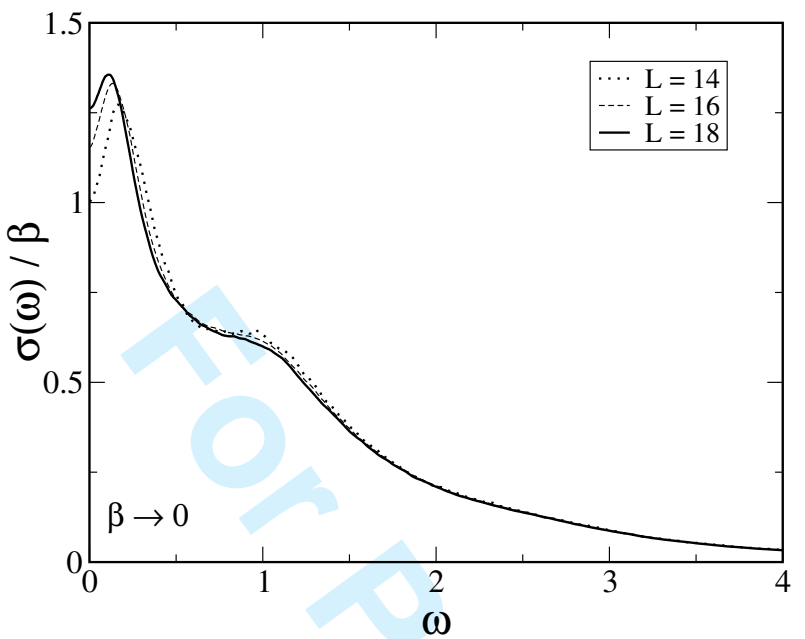

Figure 5: The spin conductivity $\sigma(\omega)$ for $\beta \rightarrow 0$.

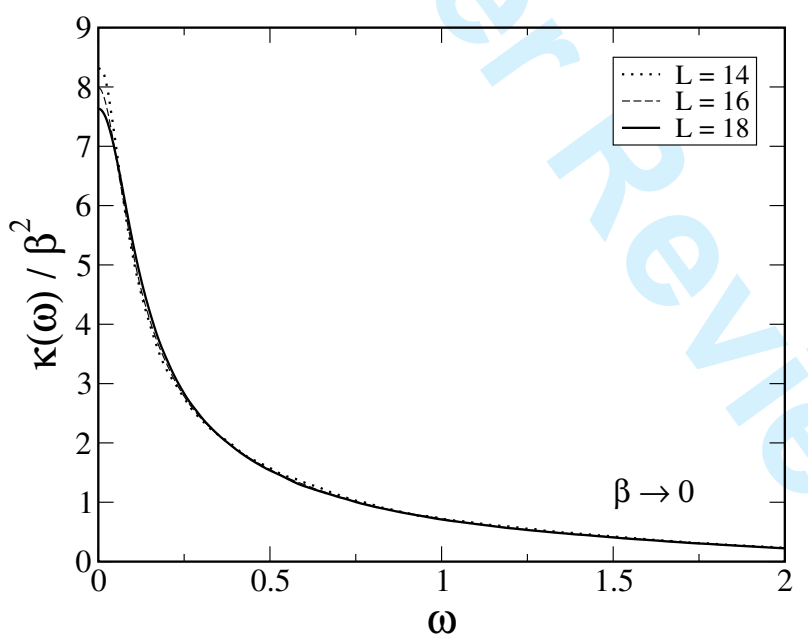

Figure 6: The thermal conductivity $\kappa(\omega)$ for $\beta \rightarrow 0$.

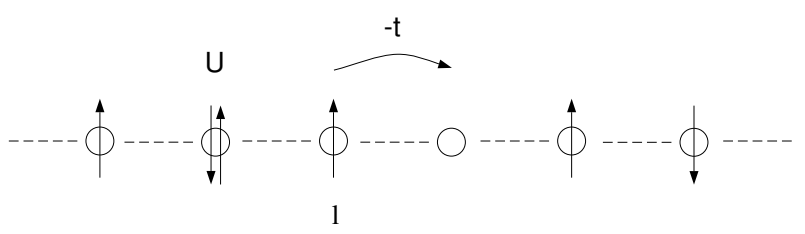

Figure 7: The Hubbard chain model 


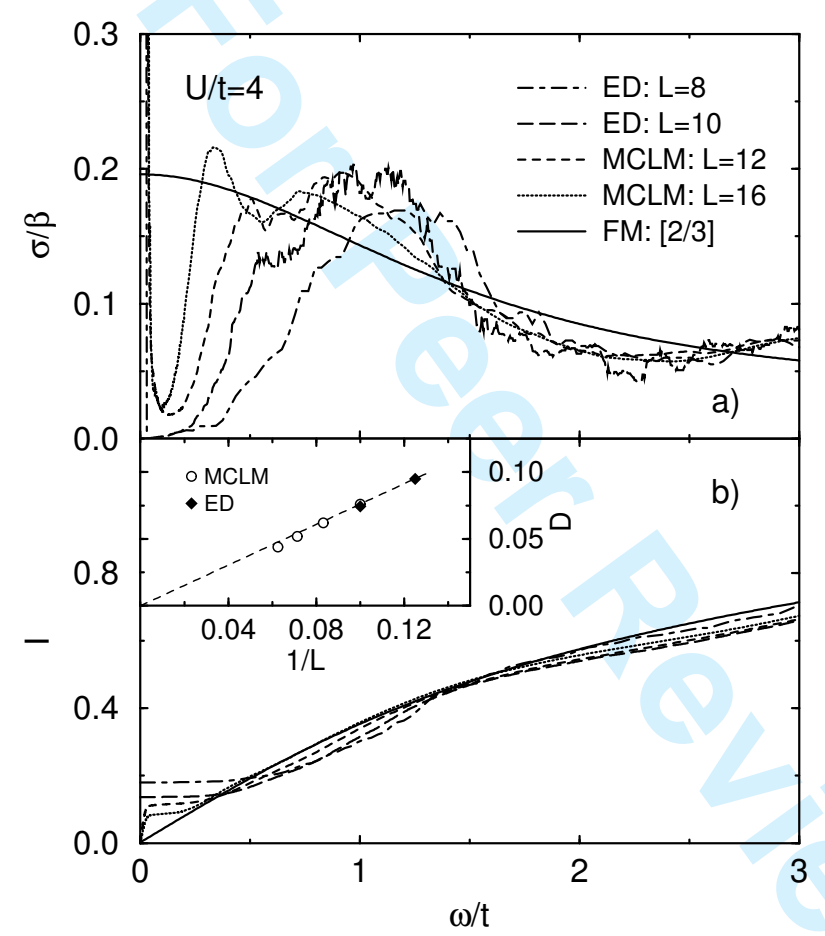

Figure 8: a) $\sigma(\omega) / \beta$ and b) $I(\omega)$ within the 1D Hubbard model with $U / t=4$, obtained via the ED, the MCLM (finite $L$ ) and a frequency moment (FM) method. The inset shows $D / \beta$ scaled vs. $1 / L$, whereby the line is guide to the eye. 